Borneo Journal of Sciences \& Technology, 4(1): 09-17

DOI: http://doi.org/10.3570/bjost.2022.4.1-03

e-ISSN: 2672-7439

(C) 2018, UTS Publisher.

Submitted: $18^{\text {th }}$ October $2021 \quad$ Accepted: $20^{\text {th }}$ December $2021 \quad$ Published: $31^{\text {st }}$ January 2022

\title{
An Update on the Viewable Mammal Species at the Natural Salt-licks in Segaliud-Lokan Forest Reserve, Sabah
}

\author{
${ }^{1}$ Lim Wing Shen, ${ }^{1}$ Andy Russel Mojiol, ${ }^{2}$ Collin Goh, ${ }^{2}$ Paul Liau and ${ }^{2}$ Sanchez Vincent John \\ ${ }^{1}$ Faculty of Tropical Forestry, Universiti Malaysia Sabah, 88400 Kota Kinabalu, Sabah, Malaysia \\ ${ }^{2}$ KTS Plantation Sdn. Bhd., Level 1, Blok 4, Jalan Utara Batu 4, 90000 Sandakan, Sabah, Malaysia
}

\begin{abstract}
A wide variety of species of terrestrial mammals are known to visit the natural salt-licks to replenish their body minerals on a regular basis, therefore indicating their suitability for mammal viewing. Natural salt-licks were discovered in Segaliud-Lokan Forest Reserve (SLFR), and then certain mammal species could be encountered at the local licks. Camera trapping survey was conducted for 8 months, to validate and update the species composition of viewable terrestrial mammals at the natural salt-licks of SLFR in this research. Four accessible natural salt-licks that situated at least $1.0 \mathrm{~km}$ from one another were selected as sampling areas. A total of 985 independent sightings of 12 different mammal species were recorded throughout 343 trap nights, where the detected individuals were comprised mainly of the large-sized ( $\mathrm{n}=948$ or $96.2 \%$ ) and threatened ( $\mathrm{n}=955$ or $97.0 \%)$ non-carnivorous ( $\mathrm{n}=982$ or $99.8 \%$ ) species that were not endemic ( $\mathrm{n}=904$ or $91.8 \%$ ) to Borneo. Different mammal species were verified to be varied in detection frequencies across different licks and times of day, and then Sambar Deer (Rusa unicolor), Bearded Pig (Sus barbatus), Bornean Orang-utan (Pongo pygmaeus), and Banteng (Bos javanicus) accounted for $95.4 \%$ ( $\mathrm{n}=940$ ) of the total number of mammalian visitations recorded in this study, thus making them the top four highly viewable species in SLFR. Although further studies are required to investigate the other natural salt-licks presented in SLFR, nevertheless this study has successfully validated the viewable mammalian composition at the given four salt-licks.
\end{abstract}

Keywords: Detection frequency, natural salt-lick, Segaliud-Lokan Forest Reserve, species composition, terrestrial mammals

\section{INTRODUCTION}

Salt-lick is a natural feature that is known to provide a wide variety of essential minerals and trace elements to the mammalian community in sufficient amounts, in the wilderness [6];[15]. However, mammal watching at the natural salt-lick is gaining popularity as one of the core attractions that are promoted at several forest protected areas in Sabah, Malaysia, which include the Deramakot Forest Reserve (DFR) and Tabin Wildlife Reserve [5];[18]. This is because the probability of sighting a large-sized mammalian individual at a natural lick is higher than other forested areas in Malaysia [2];[4]. Especially the large-sized species like the ungulates, they need to consume high amounts of sodium minerals on a regular basis, thus these species are observed at the natural salt-licks in Sabah [12];[13]. Ultimately, the suitability of a natural salt-lick for conducting the mammal watching activity has been acknowledged globally since the $20^{\text {th }}$ century [5];[10].

To date, more natural salt-licks have been found in the forest protected areas of Sabah, which includes the Segaliud-Lokan Forest Reserve (SLFR) that is located in Sandakan. Some of the local licks were investigated by past research, where the given licks were determined to be visited by 13 species of terrestrial mammals [2];[11]. However, because of the short sampling periods, particular mammal species could be missdetected from the given past studies [2];[13]. Besides that, the viewable species of terrestrial mammals, and also their respective detection frequencies, can be varied across various salt-licks and times of day, as stated by various past studies [12];[14];[15]. Therefore, this study aimed to validate the past findings of Bernard et al. [2] and Lim et al. [11], as well as to provide an update on the species composition of terrestrial mammals that can be viewed at the natural salt-licks in SLFR. The existing species composition of terrestrial mammals that can be detected at each of the examined local natural licks will be further discussed in this research.

\section{MATERIALS AND METHODS}

Sample Preparation

SLFR is a 57,247 ha permanent forest reserve, which is currently being managed by KTS Plantation Sdn Bhd as 
a Class II Commercial Forest Reserve since 1994 [22]. The 70 forest compartments in this forest reserve can be classified into Plantation Research Area (PRA), Natural Forest Management (NFM) area, Protection Area (PA), and Industrial Tree Planting (ITP) area [9]. The climate condition in SLFR is generally similar across the entire year (hot and humid), yet still the local monthly rainfall can be less than $200 \mathrm{~mm}$ during the dry season (April to September), or able to reach around $1250 \mathrm{~mm}$ during the wet season (October to March).

The four natural salt-licks that were once examined by past research [2];[11] were selected as sampling areas in this study. The selected natural licks were situated at least $1.0 \mathrm{~km}$ away from each other, thus the probability of detecting the identical individual of a certain species across different salt-licks in a short time interval (<30 min) was minimized. Nevertheless, two of the selected licks were in fact situated in the same ITP compartment (SL50_A and SL50_B), hence the given two licks were surrounded by a timber plantation forest dominated by the tree species Tandoropis (Antidesma ghaesembilla). The other two licks, on the other hand, were located in a NFM (SL59) area and a PA (SL56) area respectively. Therefore, SL56 was situated in a least-disturbed forest, while the SL59 was surrounded by a partially-disturbed forest, although the surrounding forests of these 2 licks were both the mixed lowland dipterocarp forest. Figure 1 shows the locations of the selected four natural licks in Segaliud-Lokan Forest Reserve, Sandakan, Sabah.

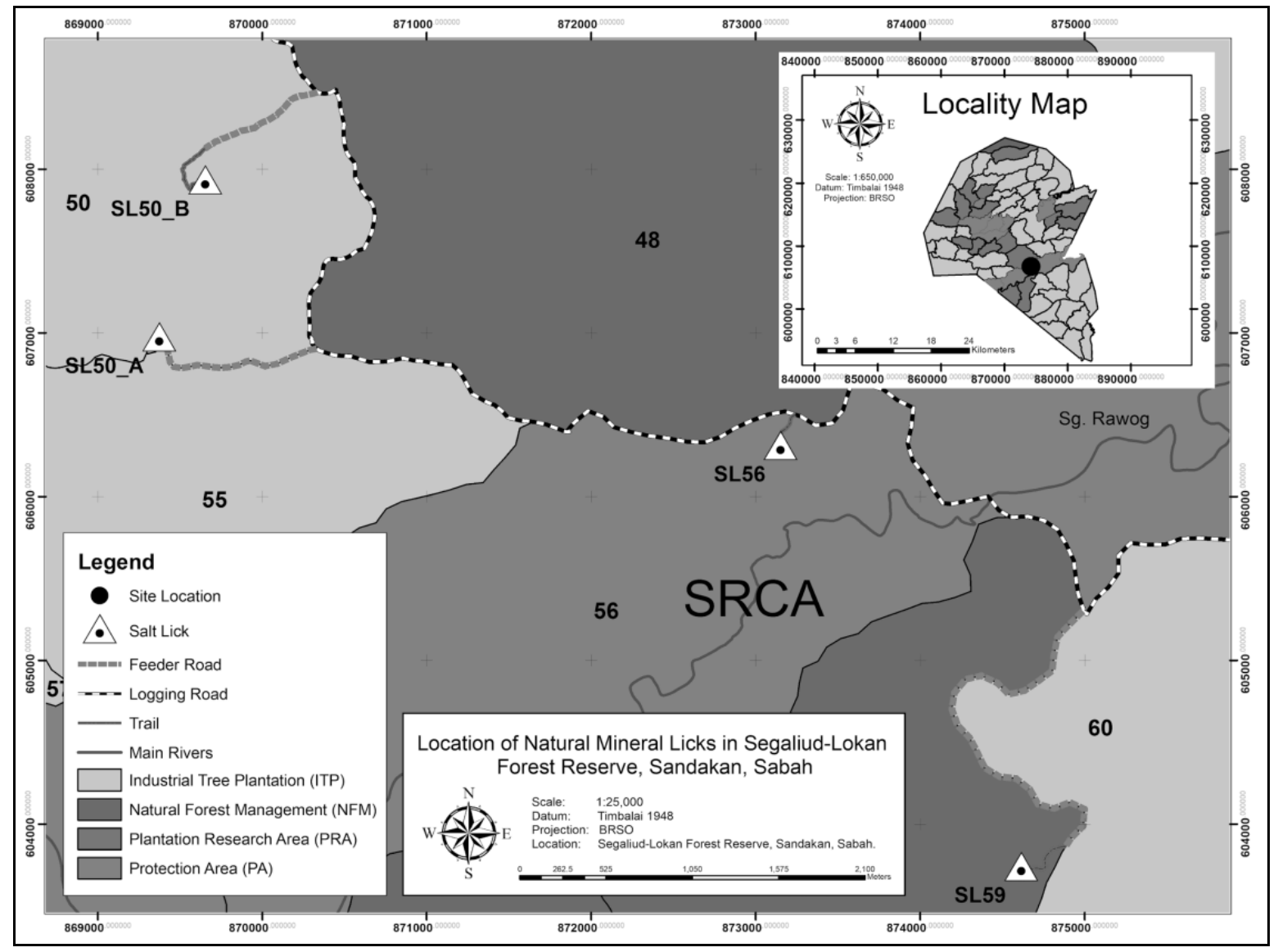

Figure 1: Locations of the selected four natural salt-licks in Segaliud-Lokan Forest Reserve, Sandakan, Sabah

\section{Data Collection}

Camera trapping survey was conducted at the selected four natural salt-licks in this study, from July 2019 until February 2020 (8 months). A unit of SunTek HC-800M camera traps equipped with the passive infra-red sensor (Hong Kong Suntek International Co., Ltd.) was placed at each selected lick (four camera traps in total), using the settings and configurations suggested by Lim et al.
[11]. The deployed camera traps were used to actively capture the footage of any medium-sized to large-sized mammalian individuals that visited the given licks, 24/7 a day through the entire sampling period of this study. Furthermore, the maintenance works (e.g.: updating the time and date, data transferring, changing batteries, and removing view-blocking bushes and shrubs) were done once per two weeks, to ensure that the deployed camera 
traps were able to function at their optimum conditions throughout the 8 months sampling period of this study.

\section{Data Analysis}

The captured photographs that without any mammalian individual were excluded from being applied in the data analysis of this study. After that, information related to the species, and also the time and date of the detection, were derived from the filtered camera trapping data for each detected mammalian individual. The species and its attributes were verified for each detected individual, by referring to relevant secondary data [1];[8];[19].

Four species traits were examined for every species of terrestrial mammals recorded in this study, including conservation status, feeding guild, endemism, and body size, as suggested by Lim et al. [11]. The body mass of an adult individual was applied in representing the body size of a particular species detected in this study, where: 1) Small < $1.0 \mathrm{~kg}$; 2) $1.0 \mathrm{~kg}<$ Medium < $45.0 \mathrm{~kg}$, and; 3) Large $>45.0 \mathrm{~kg}$ [1]. Next, the detected species were classified under three different groups of feeding guilds (omnivore, carnivore, or herbivore-frugivore), and also either as non-endemic or endemic to Borneo [11]. The conservation status for a particular detected species was represented by its existing IUCN Red List Status, which also functioned to represent its current local and global population sizes [8]. Because of that, the conservation statuses of the detected species were identified as either a least concern (LC), near-threatened (NT), vulnerable $(\mathrm{VU})$, endangered (EN), or critically endangered (CR), mammal species in this research.

The times of day used in this study were classified into the diurnal (7:01 a.m. to 5:00 p.m.), nocturnal (7.01 p.m. to 5:00 a.m.) and crepuscular (dawn: 5.01 a.m. to 7:00 a.m.; Dusk: 5:01 p.m. to 07:00 p.m.) hours, based on Ikeda et al. [7]. After that, the detection frequency of a certain mammal species at a given lick and time was represented by relative abundance index (RAI) in this study. Henceforth, RAI was calculated as the number of independent detection rate of a certain mammal species at a specific lick or time, per 100 trap nights $\left(100 \mathrm{TN}^{-1}\right)$ [11], by using the formula as shown in below:

$$
\mathrm{RAI}_{\mathrm{ij}}=\left(\mathrm{n}_{\mathrm{ij}} / \mathrm{TN}_{\mathrm{j}}\right) \times 100
$$

Where, $\mathrm{RAI}_{\mathrm{ij}}$ is the relative abundance index of species $i$ at condition $j$, in which $j$ can be referred to a particular lick, time, or overall; $\mathrm{n}_{\mathrm{ij}}$ is the independent detection of species $\mathrm{i}$ at condition $\mathrm{j}$, and; $\mathrm{TN}_{\mathrm{j}}$ is the total trap night at condition $\mathrm{j}$.

The viewable mammalian compositions at different salt-licks were determined using Chi-Square test. Then, species rank abundance curves were plotted to ascertain the ranking of every recorded mammal species, in terms of their respective detection frequencies, at the selected four licks in this research. The temporal activity pattern was identified descriptively for the detected species, by referring to the detection frequencies of a given species at the selected four natural salt-licks throughout various times of day. The Chi-Square test was derived using the "chisq.test" function from the "MASS" package, in the statistical software RStudio ver. 1.2.5001 [20];[21], with the confidence interval level of this research was set at $95.0 \%(\mathrm{p}=0.05)$.

\section{RESULTS}

Twelve different species of terrestrial mammals, which belonged to five different orders and nine different families, were recorded for 985 times independently in 343 trapping nights at the selected four natural salt-licks in this research. The Artiodactyla order was determined with the highest number of detected species, namely the Banteng (Bos javanicus) from Bovidae family, Bearded Pig (Sus barbatus) from Suidae family, Sambar Deer (Rusa unicolor) from Cervidae family, and Mousedeer (Tragulus spp.) from Tragulidae family. Both species of the mousedeer (T. kanchil and T. napu) were difficult to distinguish from each other when referring towards the captured footage, therefore both species were identified as Mousedeer (Tragulus spp.) in this research. Moving on, the Primate order was comprised of three species, in which both the Red Leaf Monkey (Presbytis rubicunda) and the Pig-tailed Macaque (Macaca nemestrina) were belonged to the Cercopithecidae family, while Bornean Orang-utan (Pongo pygmaeus) was from the Hominidae family. Then, the Common Palm Civet (Paradoxurus hermaphroditus) and Malay Civet (Viverra tangalunga) were under the Carnivora order and Viverridae family, while the Thick-spined Porcupine (Hystrix crassispinis) and Malayan Porcupine (H. brachyura) were both from the Rodentia order and Hystricidae family in this study. Lastly, the Bornean Pygmy Elephant (Elephas maximus borneensis) was in fact the sole species belonging to the Proboscidae order and Elephantidae family, recorded in this study. Table 2 shows the list of species of terrestrial mammals detected at the selected four natural salt-licks in SLFR.

The species composition of mammalian individuals detected at the selected four licks in SLFR was verified using the Chi-Squared test. The results of this analysis revealed that the detected mammalian individuals were comprised majorly of the threatened ( 7 species; $\mathrm{n}=955$ or $97.0 \% ; \chi^{2}{ }_{9}=150.8, \mathrm{p}<0.001$ ), large-sized (6 species; $\mathrm{n}=948$ or $\left.96.2 \% ; \chi^{2}{ }_{3}=53.1, \mathrm{p}<0.001\right)$, non-endemic $(8$ species; $\mathrm{n}=904$ or $91.8 \%$; $\left.\chi^{2}{ }_{3}=116.03, \mathrm{p}<0.001\right)$, and non-carnivorous species ( 10 species; $\mathrm{n}=982$ or $99.8 \%$; $\left.\chi^{2}{ }_{6}=108.2, p<0.001\right)$, especially at the SL59, SL56 and SL50_B $(\mathrm{p}<0.001)$ in SLFR. Similarly, the given types of mammal species were detected most frequently at the SL50_A as well $(\mathrm{p}<0.001)$. Nonetheless, the difference 
in detection frequency between the omnivorous species herbivorous-frugivorous species was insignificant $\left(\chi^{2}{ }_{1}=\right.$ 2.3901, $\mathrm{p}=0.122$ ), although the herbivorous-frugivorous species was detected generally more frequent $(\mathrm{n}=60$ or $73.2 \%)$ than the omnivorous species $(n=22$ or $26.8 \%)$ at the given lick in this study.

Moving on, certain mammal species were detected more frequently at the natural salt-licks in SLFR during a specific time of day. Hence, the detection frequencies of the 12 recorded species were determined to be varied across different times in this study, as shown in below Figure 2. Among the recorded species, H. brachyura, V. tangalunga and $P$. hermaphroditus were detected only at night, whereas both $M$. nemestrina and $P$. rubicunda were recorded only during daytime in this study $(100.0$ $\%)$. E. maximus majorly visited the local licks at night (75.0\%), while the Tragulus spp. visited the given licks similarly frequent throughout the day.

Table 1: A list of species of terrestrial mammals detected at the selected four natural salt-licks in SLFR

\begin{tabular}{|c|c|c|c|c|c|}
\hline Species & $\begin{array}{l}\text { Feeding } \\
\text { Guild }\end{array}$ & Endemism & $\begin{array}{l}\text { IUCN Red } \\
\text { List Status }\end{array}$ & $\begin{array}{l}\text { Body Size } \\
\text { (Category) }\end{array}$ & $\begin{array}{c}\text { RAI } \\
\left(100 \mathrm{TN}^{-1}\right)\end{array}$ \\
\hline \multicolumn{6}{|l|}{ Artiodactyla Order } \\
\hline $\begin{array}{l}\text { Bovidae Family } \\
\text { Banteng (Bos javanicus) }\end{array}$ & $\mathrm{HF}$ & No & $\mathrm{EN}$ & Large & 14.87 \\
\hline $\begin{array}{l}\text { Cervidae Family } \\
\text { Sambar Deer (Rusa unicolor) }\end{array}$ & $\mathrm{HF}$ & No & VU & Large & 152.8 \\
\hline $\begin{array}{l}\text { Suidae Family } \\
\text { Bearded Pig (Sus barbatus) }\end{array}$ & $\mathrm{O}$ & No & VU & Large & 90.67 \\
\hline $\begin{array}{l}\text { Tragulidae Family } \\
\text { Mousedeer (Tragulus spp.) }\end{array}$ & $\mathrm{HF}$ & No & $\mathrm{LC}$ & Medium & 2.915 \\
\hline \multicolumn{6}{|l|}{ Primates Order } \\
\hline $\begin{array}{l}\text { Hominidae Family } \\
\text { Bornean Orang-utan (Pongo pygmaeus) }\end{array}$ & $\mathrm{O}$ & Yes & $\mathrm{CR}$ & Large & 15.74 \\
\hline $\begin{array}{l}\text { Cercopithecidae Family } \\
\text { Pig-tailed Macaque (Macaca nemestrina) } \\
\text { Red Leaf Monkey (Presbytis rubicunda) }\end{array}$ & $\begin{array}{c}\mathrm{O} \\
\mathrm{HF}\end{array}$ & $\begin{array}{l}\text { No } \\
\text { Yes }\end{array}$ & $\begin{array}{l}\text { EN } \\
\text { VU }\end{array}$ & $\begin{array}{l}\text { Medium } \\
\text { Medium }\end{array}$ & $\begin{array}{l}1.166 \\
0.875\end{array}$ \\
\hline \multicolumn{6}{|l|}{ Rodentia Order } \\
\hline $\begin{array}{l}\text { Hystricidae Family } \\
\text { Malayan Porcupine (Hystrix brachyura) } \\
\text { Thick-spined Porcupine (Hystrix crassispinis) }\end{array}$ & $\begin{array}{l}\mathrm{O} \\
\mathrm{O}\end{array}$ & $\begin{array}{l}\text { No } \\
\text { Yes }\end{array}$ & $\begin{array}{l}\mathrm{LC} \\
\mathrm{LC}\end{array}$ & $\begin{array}{l}\text { Medium } \\
\text { Medium }\end{array}$ & $\begin{array}{l}0.583 \\
4.665\end{array}$ \\
\hline \multicolumn{6}{|l|}{ Proboscidae Order } \\
\hline $\begin{array}{l}\text { Elephantidae Family } \\
\text { Bornean Pygmy Elephant } \\
\text { (Elephas maximus borneensis) }\end{array}$ & $\mathrm{HF}$ & Yes & EN & Large & 2.915 \\
\hline \multicolumn{6}{|l|}{ Carnivora Order } \\
\hline $\begin{array}{l}\text { Viverridae Family } \\
\text { Common Palm Civet } \\
\text { (Paradoxurus hermaphroditus) }\end{array}$ & $\mathrm{C}$ & No & $\mathrm{LC}$ & Medium & 0.292 \\
\hline
\end{tabular}

*Note: $\mathrm{C}=$ Carnivore; $\mathrm{HF}=$ Herbivore-Frugivore; $\mathrm{O}=$ Omnivore; $\mathrm{LC}=$ Least Concern; $\mathrm{VU}=\mathrm{Vulnerable}$; EN = Endangered; $\mathrm{CR}=$ Critically Endangered, and; RAI $=$ Relative Abundance Index. 


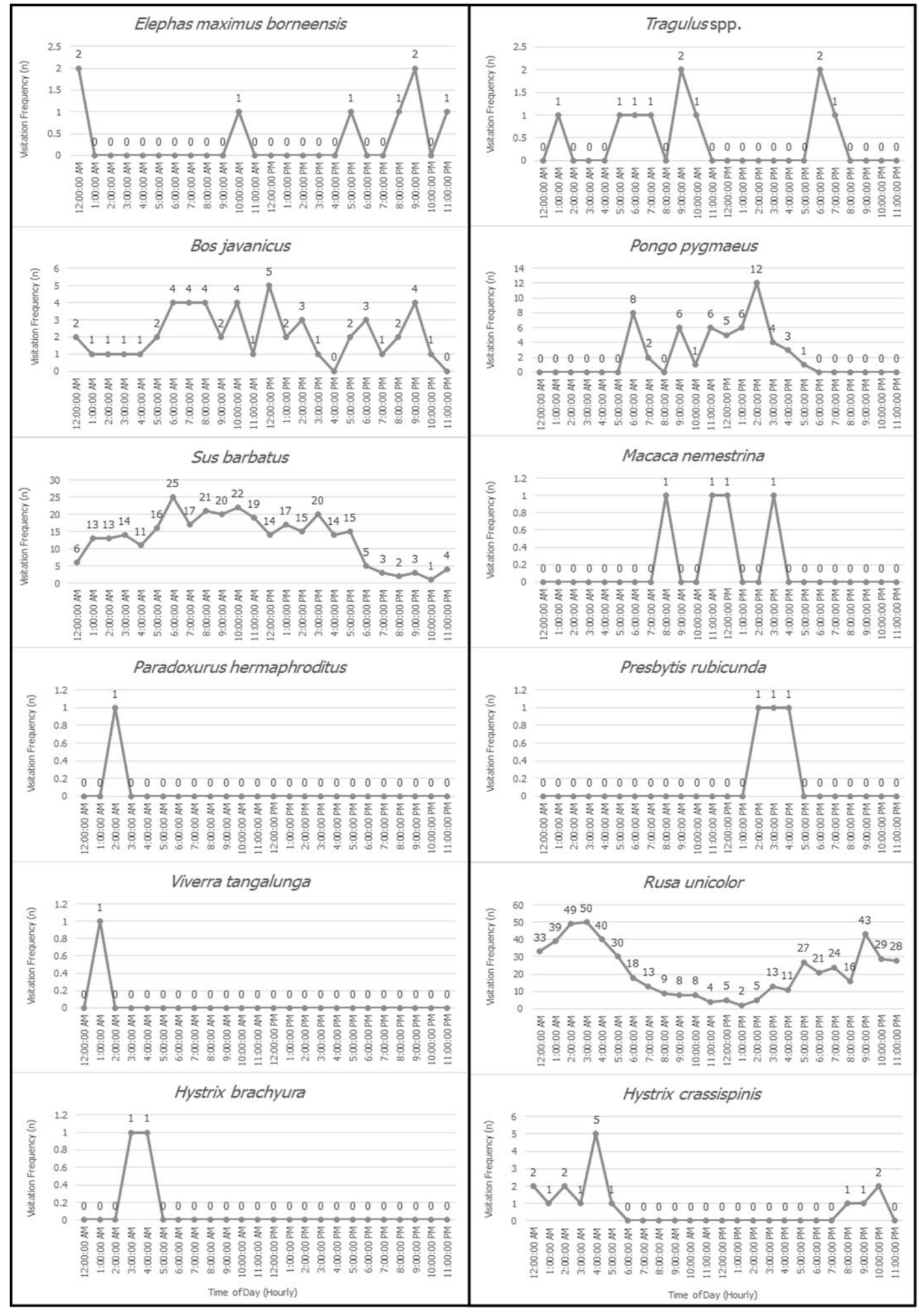

Figure 2: Detection frequencies of the twelve detected species of terrestrial mammals across different times of day, at the selected four natural salt-licks in Segaliud-Lokan Forest Reserve. 
Both $R$. unicolor and H. crassispinis were detected at the local salt-licks very frequently, especially during the late night hours $(R$. unicolor: $\mathrm{n}=351$ or $66.9 \% ; H$. crassispinis: $\mathrm{n}=15$ or $93.75 \%$ ). Both $S$. barbatus and $B$. javanicus, on the other hand, visited the given four licks generally most frequent at daytime (S. barbatus: $\mathrm{n}=179$ or $57.7 \% ; B$. javanicus: $\mathrm{n}=26$ or $51.0 \%)$. P. pygmaeus was inactive at night $(\mathrm{n}=0)$, and thus this species mainly visited the given licks during daytime as well $(\mathrm{n}=45$ or $83.3 \%)$, although at different time ( $P$. pygmaeus: 2.00 p.m.) with both the $B$. javanicus $(12.00$ p.m.) and $S$. barbatus (8.00 a.m. to 10.00 a.m.). In other words, both the $H$. crassispinis and $R$. unicolor were determined as nocturnal species, while both $S$. barbatus, $P$. pygmaeus, and $B$. javanicus were verified as diurnal species, based on their respective detection frequencies at the selected four natural salt-licks in SLFR, in this study.
The species rank abundance curves were plotted to display the mammalian detection frequencies at the four examined salt-licks, as shown in below Figure 3. Based on Figure 3, R. unicolor was determined to be detected most frequently $\left(\mathrm{RAI}=152.8100 \mathrm{TN}^{-1}\right)$, and followed by $S$. barbatus $\left(\mathrm{RAI}=90.67100 \mathrm{TN}^{-1}\right)$, . pygmaeus $(\mathrm{RAI}=$ $\left.15.741_{100 \mathrm{TN}^{-1}}\right)$, B. javanicus $\left(\mathrm{RAI}=14.87100 \mathrm{TN}^{-1}\right), H$. crassispinis $\left(\mathrm{RAI}=4.67100 \mathrm{TN}^{-1}\right)$, Tragulus $\mathrm{spp}$. $(\mathrm{RAI}=$ $\left.2.92100 \mathrm{TN}^{-1}\right)$, E. maximus $\left(\mathrm{RAI}=2.332100 \mathrm{TN}^{-1}\right), M$. nemestrina $\left(\mathrm{RAI}=1.166100 \mathrm{TN}^{-1}\right), P$. rubicunda $(\mathrm{RAI}=$ $\left.0.875100 \mathrm{TN}^{-1}\right), H$. brachyura $(\mathrm{RAI}=0.583100 \mathrm{TN}-1)$, and finally both $V$. tangalunga and $P$. hermaphroditus (RAI $=0.292100 \mathrm{TN}^{-1}$ ) in this study. The given findings highlighted that both $R$. unicolor and $S$. barbatus were detected about once per day, and then both $P$. pygmaeus and $B$. javanicus were recorded once a week, while the remaining eight species were rarely being detected (less than once a month), at the given four licks in this study.

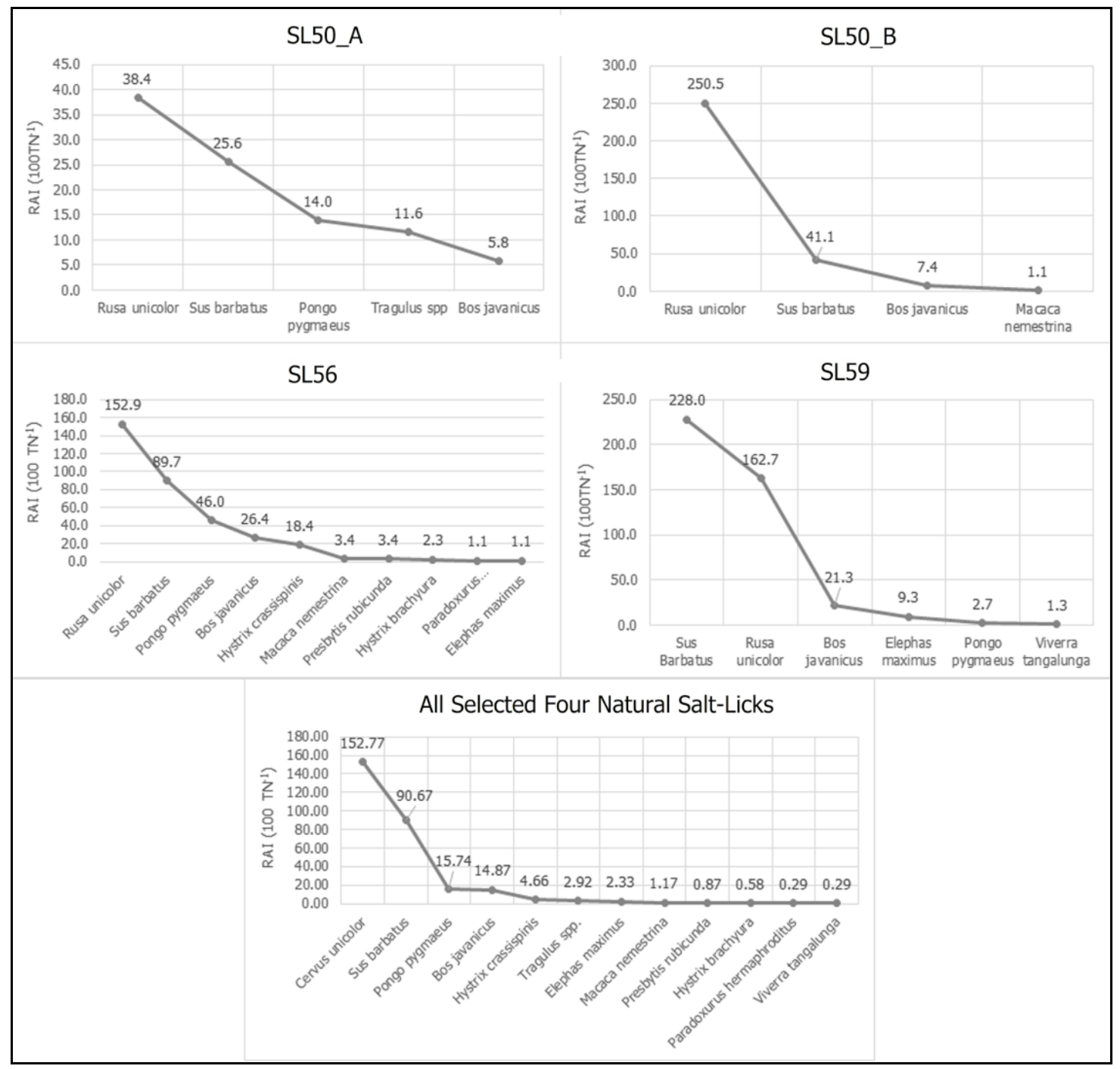

Figure 3: Species rank abundance curves for the twelve detected species of terrestrial mammals, by according to their respective detection frequencies (RAIs) in a decreasing order at the selected four natural salt-licks 
Likewise, $R$. unicolor was detected most frequently at SL50_A (RAI=34.8 $\left.100 \mathrm{TN}^{-1}\right)$, SL50_B (RAI=250.5 $\left.100 \mathrm{TN}^{-1}\right)$ and SL56 $\left(\mathrm{RAI}=152.9100 \mathrm{TN}^{-1}\right)$, except at the SL59 (RAI=162.7 $\left.100 \mathrm{TN}^{-1}\right)$, because $S$. barbatus was detected most frequently at SL59 (RAI=228.0 $100 \mathrm{TN}^{-}$ $\left.{ }^{1}\right)$. At the same time, $S$. barbatus was detected secondmost often at SL50_A (RAI=25.58 $\left.100 \mathrm{TN}^{-1}\right)$, SL50_B $\left(\mathrm{RAI}=41.05100 \mathrm{TN}^{-1}\right)$, and SL56 $\left(\mathrm{RAI}=89.65100 \mathrm{TN}^{-}\right.$ $\left.{ }^{1}\right)$ in this study. Next, P. pygmaeus was detected thirdmost often at both SL50_A (RAI=13.95 $100 \mathrm{TN}^{-1}$ ) and SL56 (RAI $=45.98100 \mathrm{TN}^{-1}$ ), but then second-least frequently at SL59 $\left(\mathrm{RAI}=2.667100 \mathrm{TN}^{-1}\right)$. Additionally, P. pygmaeus was failed to be detected at SL50_B, thus indicating that this primate species did not visit SL50_B throughout the 8 months sampling period of this study.

Furthermore, B. javanicus was detected third-most frequently at both SL50_B (RAI=7.368 $100 \mathrm{TN}^{-1}$ ) and SL59 (RAI=21.3 $\left.100 \mathrm{TN}^{-1}\right)$, and fourth-most frequently at the SL56 $\left(\mathrm{RAI}=26.44100 \mathrm{TN}^{-1}\right)$, and least frequently at SL50_A (RAI=5.814 100 $\left.\mathrm{TN}^{-1}\right)$. Tragulus spp., on the other hand, was detected second-least frequently at the SL50_A (RAI=11.63 100TN-1), because the detection frequencies of the two Mousedeer species were pooled together in this study. Since the detection frequencies of the given two Mousedeer species to SL50_A remain uncertain, thus the results of this research indicated that both species of Mousedeer could only be encountered at SL50_A in SLFR, throughout the sampling period.

A total of four species of terrestrial mammals were detected in low frequencies, yet only at SL56, including $P$. hermaphroditus $\left(\mathrm{RAI}=1.149100 \mathrm{TN}^{-1}\right), P$. rubicunda $\left(\mathrm{RAI}=3.45100 \mathrm{TN}^{-1}\right), H$. brachyura $\left(\mathrm{RAI}=2.30100 \mathrm{TN}^{-}\right.$ ${ }^{1}$ ), and $H$. crassispinis $\left(\mathrm{RAI}=18.39100 \mathrm{TN}^{-1}\right)$. As for $V$. tangalunga, this species was detected once only at the SL59 $\left(\mathrm{RAI}=1.333100 \mathrm{TN}^{-1}\right)$. Then, M. nemestrina was detected the least at SL50_B (RAI=1.053 100 $\left.\mathrm{TN}^{-1}\right)$ and sixth-most frequently at SL56 (RAI=3.448 $100 \mathrm{TN}^{-1}$ ), while E. maximus was the third-least detected species at SL59 $\left(\mathrm{RAI}=9.333100 \mathrm{TN}^{-1}\right)$ and least detected species at SL56 (RAI=1.15 100 $\mathrm{TN}^{-1}$ ). In summary, $R$. unicolor, $S$. barbatus, $P$. pygmaeus, and B. javanicus visited the selected four natural salt-licks in SLFR generally more frequently than the remaining eight species of terrestrial mammals detected in this study. Actually, the detection frequencies of these four elusive large-sized threatened species accounted for about $95.4 \%(n=940)$ of the total number of mammalian detections that were recorded in this study, thus making these species the top four highly viewable species at the given four licks in SLFR.

\section{DISCUSSION}

The finding of this research suggested that 12 different species of terrestrial mammals were detected at the four selected natural salt-licks, throughout eight months in SLFR. The twelve species recorded in this study were in fact identical to the mammal species reported by Lim et al. [11], hence emphasizing that no additional species was detected at the given four licks in this study. Next, this study managed to cover about $88.9 \%$ of the species detected by Bernard et al. [2] at SL50_A in SLFR, 11 species $(37.9 \%)$ that used the natural licks at DFR [13], and five species $(71.4 \%$ ) observed by Hon and Shibata [6] at the natural salt-licks situated in Anap Sustainable Development Unit (ASDU). Likewise, Sunda Pangolin Pangolin (Manis javanica) was reported using SL50_A [2], and then the Bornean Yellow Muntjac (Muntiacus atherodes) could be observed at both ASDU and DFR [6];[12];[13]. Even though $M$. atherodes was not detected visiting the salt-licks at SLFR by past research, still the presence of this ungulate species in SLFR was validated by Wilting and Azlan [22]. P. rubicunda, on the other hand, was detected at the natural salt-lick in Borneo for the first time in this research. Since this primate species could obtain essential minerals in sufficient amounts by consuming termite nests, thus $P$. rubicunda exhibit low lick dependency, and subsequently resulting in its low detection frequency recorded at SL56 in this study [19].

Variability in the recorded species number between this study and the 3 previously mentioned past research were likely to be caused by the difference in sampling efficiency, in terms of the number of deployed camera traps per lick, and also the sampling period used for the camera trapping survey. Matsubayashi et al. [13] used both field observation and camera trapping techniques, to determine the mammal species that visited the five natural salt-licks presented in DFR for 13 months. Hon and Shibata [6], on the other hand, applied only camera trapping survey in investigating this matter at three saltlicks for 8 months, which was similar to that of Bernard et al. [2] that was conducted at SL50_A for 7 months, and also to this study. At the same time, this study only deployed one camera trap per lick, whereas these three past studies deployed two camera traps per salt-lick, for enhancing the detection probabilities of the individuals of terrestrial mammals [16]. Henceforth, the conducting of direct observation survey inside and outside the saltlicks, and also the increasing in sampling period and the number of deployed camera trap per lick, can be used in explaining the higher species number recorded by past studies [12];[13], as well as the variability in number of species recorded by both Bernard et al. [2] and Hon and Shibata [6], when compared to those of this study.

Next, the non-endemic, threatened, large-sized, and herbivorous-frugivorous species used the selected four natural salt-licks in SLFR most frequently, although the omnivorous species also used the given licks frequently throughout the sampling period of this study. The given findings were aligned with the findings of several past studies [2];[6];[12];[13], indicating that the given mammal species are highly dependent on the natural salt-licks in Borneo, majorly for mineral consumption 
on a regular basis. Besides that, these species used the selected four licks in SLFR very frequently, except for SL50_A. This emphasizes that the local terrestrial mammals avoid using the salt-licks that are located near to the roads and surrounded by disturbed forest habitats, mostly to avoid overlapping with the human activity pattern at SL50_A [2];[12]. Henceforth, these species mainly visited SL59 that was located far from the road, and at the same time situated in a partially-disturbed forest, which further the fact that surrounding environments have certain impacts to the detection frequency of a certain mammal species at a given saltlick in this forest reserve [10];[14].

The detection frequencies of the twelve species of terrestrial mammals recorded in this study were verified to be varied across different licks throughout this study. Actually, only R. unicolor, S. barbatus and B. javanicus were detected at all of the selected four salt-licks, while the remaining nine species failed to be detected at certain licks in SLFR. This phenomenon can be resulted from the response of a mammal species upon both the onsite and surrounding conditions of a salt-lick, where this species avoid using the salt-licks with unfavourable conditions, and vice versa for the licks with favourable conditions [13];[14]. Especially P. pygmaeus, the given primate species showed higher preference in inhabiting the least-disturbed forests and undisturbed forest than the disturbed natural forests and plantation forests [12]. Henceforth, each detected mammal species were ranked differently across the given four licks, in terms of their respective detection frequencies, in this study.

Other than that, only the $R$. unicolor, S. barbatus $P$. pygmaeus, and B. javanicus were detected frequently at the selected four natural licks, throughout this research. The given large-sized and threatened species comprised a majority of the mammalian detections recorded in this study ( $\mathrm{n}=940$ or $95.4 \%$ ), mostly because these elusive species were required to visit the given licks for mineral consumptions regularly [2];[12];[14]. Nevertheless, the $R$. unicolor was detected most frequently during nocturnal hours, although it was detected frequently at daytime as well, and vice versa for S. barbatus and $B$. javanicus in this study. On the contrary, P. pygmaeus was detected mainly during daytime, as the primate species increased their activity levels when the sun rose, and vice versa as the sun set [19]. Likewise, both the $R$. unicolor and $H$. crassispinis were detected majorly during late night, but at different times $(R$. unicolor: 2.00 a.m. to 3.00 a.m.; H. crassispinis: 4.00 a.m.). Similarly, the B. javanicus, S. barbatus and $P$. pygmaeus frequently used the given licks at different times during the diurnal hours as well (B. javanicus: 12.00 p.m.; P. pygmaeus: 2.00 p.m.; S. barbatus: 8.00 a.m. to 10.00 a.m.). The given variations in peak visiting hour among these mammal species can be resulted from the temporal niche partitioning, which is adopted by a given species to avoid the risks of being predated and hunted, and also interspecific competition, with other terrestrial mammal species [6, 7]. Regarding the remaining seven species, their sample sizes were small, since they were seldom being detected $(n<10)$ at these natural licks. Therefore, their respective temporal activity patterns in visiting the local salt-licks failed to be determined in this research. However, Matsubayashi et al. [13] highlighted that these seven species seldom visited the natural licks at DFR as well, thus indicating that the detections of these species were difficult, unlike those large-sized and threatened species, at the natural salt-licks in Sabah [4].

In summary, the elusive $R$. unicolor, $P$. pygmaeus, $S$. barbatus, and B. javanicus were determined to be the top four highly viewable species of terrestrial mammals at the selected four natural salt-licks in SLFR. Still, this study was conducted onto the four natural salt-licks that were investigated by past studies [2];[11]. Since both the onsite and surrounding conditions can be varied among different licks, hence the detected mammal species can be different between the given licks, which are situated in the same forest area [14];[15]. This phenomenon can explain the miss-detection of both $M$. atherodes and M. javanica, as well as other mammal species that inhabit the forest of SLFR, in this research [9];[22]. Henceforth, there is a need to conduct further study on the species composition of the viewable terrestrial mammals at the other natural salt-licks presented in SLFR in future.

\section{CONCLUSION}

This study has recorded 12 different species of medium to large-sized terrestrial mammals that can be observed at four natural salt-licks (SL50_A, SL50_B, SL56, and SL59) in SLFR. Among these twelve recorded species, $R$. unicolor can be observed most frequently at the local salt-licks, and followed by S. barbatus, $P$. pygmaeus, $B$. javanicus, $H$. crassispinis, Tragulus spp., E. maximus, M. nemestrina, P. rubicunda, H. brachyura, and finally both $V$. tangalunga, and $P$. hermaphroditus. After that, the elusive $R$. unicolor, S. barbatus, P. pygmaeus, and $B$. javanicus visit the local natural licks at least once a week, while the remaining eight species visit the given four licks only once per month. Henceforth, these four large-sized species are identified as the top four highly viewable species of terrestrial mammals at the natural salt-licks in this forest reserve. Additionally, a majority of the mammalian individuals that visit the given licks are in fact the threatened, non-endemic, large-sized, and herbivorous-frugivorous species. Next, different species mainly visit the given natural licks at different times of day, where $S$. barbatus, $P$. pygmaeus and B. javanicus are verified as diurnal species, whereas $R$. unicolor and $H$. crassispinis are determined to be nocturnal species. Since this research has been conducted only on the four 
salt-licks examined by past studies, therefore there is a possibility that certain mammal species that inhabit the forest habitat of SLFR have been miss-detected. Hence, future research should be conducted into this matter, at the other salt-licks presented in this forest reserve. Still, the findings of this research are able to validate those of the relevant past studies, therefore the objective of this study has been achieved.

\section{ACKNOWLEDGEMENT}

The authors would like to offer their sincere gratitude to both the KTS Plantation Sdn. Bhd., and Sabah Forestry Department, for permission and assisting the authors in conducting the research in SLFR. This research has been funded by the External Collaboration Research Grant (GKP0023-2018), and also UMSGreat Research Grant (GUG0322-1/2019).

\section{REFERENCES}

[1] Andrews, P., J. M. Lord and E. M. Nesbit Evans, 1979. Patterns of ecological diversity in fossil and modern mammalian faunas. Biological Journal of the Linnean Society, 11: 177-205.

[2] Bernard, H., N. Joseph, A. H. Ahmad, S. L. Kee, J. Sompud, M. Nakabayashi, R. Nilus, M. A. F. Suis, H. Muin, R. Jaikim, A. Md. Yakub, A. Jamali, M. Anson, E. Alim, P. Liau \& C. Goh, 2019. An assessment of the terrestrial mammal community in and around Sungai Rawog Conservation Area, Sabah, Malaysia. Proceeding of the Seminar on Sungai Rawog Conservation Area Scientific Expedition. Sabah Forestry Department and KTS Plantation Sdn. Bhd., Feb. 21, pp: 137-158.

[3] Blake, J. G., D. Mosquera and J. Salvador, 2013. Use of mineral licks by mammals and birds in hunted and non-hunted areas of Yasuní National Park, Ecuador. Animal Conservation, 16(4): 430-437.

[4] Chew, M. Y., K. Hymeir, R. Nosrat and M. A. Shahfiz, 2014. Relation between grasses and large herbivores at the Ulu Muda salt licks, Peninsular Malaysia. Journal of Tropical Forest Science, 26(4): 554-559.

[5] Chong, M. H. N., S. H. Tang and S. Suksuwan, 2005. Management Recommendations for Wildlife Saltlicks with Particular Reference to Sira Air Hangat at Ulu Muda Forest Reserve, Kedah. WWFMalaysia, Selangor.

[6] Hon, J. and S. Shibata, 2013. Temporal Partitioning by Animals Visiting Salt Licks. International Journal of Environmental Science and Development, 4(1): 44-48.

[7] Ikeda, T., K. Uchida, Y. Matsuura, H. Takahashi, T. Yoshida, K. Kaji and I. Koizumi, 2016. Seasonal and diel activity patterns of eight sympatric mammals in northern Japan revealed by an intensive camera-trap survey. PLoS ONE, 11(10): 1-16.

[8] International Union for Conservation of Nature and Natural Resources. 2022. The IUCN Red List of
Threatened Species. Retrieved from http://www.iucnredlist.org. Accessed on 15/10/21.

[9] Kee, S. L., J. Sompud, K. J. C. Pei, M. Sudin, C. Goh, P. Liau and F. Yahya, 2018. Nocturnal Mammals of Segaliud-Lokan Forest Reserve, Sabah. Transactions on Science and Technology, 5(2): 131136.

[10] Lim, W. S. and A. R. Mojiol, 2020. A review of mammalian availability as salt-lick tourism supply. Borneo Journal of Sciences and Technology, 2 (1): $32-40$.

[11] Lim, W. S., P. M. Yip, A. R. Mojiol, C. Goh, P. Liau, Y. C. Liaw and S. V. John. 2020. Feasibility of natural salt-licks for wildlife-watching in SegaliudLokan Forest Reserve, Sandakan, Sabah. IOP Conference Series: Earth and Environmental Science, 549 (1): 1-8.

[12] Matsubayashi, H., A. H. Ahmad, N. Wakamatsu, E. Nakazono, M. Takyu, N. Majalap, P. Lagan and J. R. A. Sukor, 2011. Natural-licks use by orangutans and conservation of their habitats in Bornean tropical production forest. Raffles Bulletin of Zoology, 59(1): 109-115.

[13] Matsubayashi, H., P. Lagan, N. Majalap, J. Tangah, J. R. A. Sukor and K. Kitayama, 2007. Importance of natural licks for the mammals in Bornean inland tropical rain forests. Ecological Research, 22(5): 742748.

[14] Matsuda, I., M. Ancrenaz, Y. Akiyama, A. Tuuga, N. Majalap and H. Bernard, 2015. Natural licks are required for large terrestrial mammals in a degraded riparian forest, Sabah, Borneo, Malaysia. Ecological Research, 30(1): 191-195.

[15] Molina, E., T. E. León and D. Armenteras, 2014. Characteristics of natural salt licks located in the Colombian Amazon foothills. Environmental Geochemistry and Health, 36(1): 117-129.

[16] Pease, B. S., C. K. Nielsen and E. J. Holzmueller, 2016. Single-camera trap survey designs miss detections: Impacts on estimates of occupancy and community metrics. PLoS ONE, 11(11): 1-14.

[18] Peters, R. F. and L. E. Min, 2018. The Role of Wildlife-viewing Activity at Tabin Wildlife Reserve. Journal of Tropical Biology and Conservation, 15: 29-41.

[19] Phillips, Q. \& K. Phillips, 2018. Phillips' Field Guide to the Mammals of Borneo and Their Ecology: Sabah, Sarawak, Brunei and Kalimantan. 2nd Edn., John Beaufoy Publishing Ltd., Oxford.

[20] R Core Team. 2022. R: A language and environment for statistical computing. R Foundation for Statistical Computing, Vienna.

[21] Venables, W. N. \& B. D. Ripley, 2002 Modern Applied Statistics with S. 4th Edn., Springer, New York.

[22] Wilting, A. and Azlan, 2010. Wildlife surveys in Segaliud Lokan Forest Reserve, KTS Plantations. Final report. Conservation of Carnivores in Sabah, Sabah Forestry Department, Sabah. 